\title{
Intensive Pre-Processing of KDD Cup 99 for Network Intrusion Classification Using Machine Learning Techniques
}

\author{
https://doi.org/10.3991/ijim.v13i01.9679 \\ Ibrahim M. Obeidat ${ }^{(\varpi)}$, Nabhan Hamadneh \\ Hashemite University, Az-Zarqa, Jordan \\ imsobeidat@hu.edu.jo \\ Mouhammd Alkasassbeh, \\ Princess Sumaya University for Technology, Amman, Jordan. \\ Mohammad Almseidin \\ University of Miskolc, Miskolc, Hungary \\ Mazen Ibrahim AlZubi \\ Zayed University, Dubai, United Arab Emirates
}

\begin{abstract}
Network security engineers work to keep services available all the time by handling intruder attacks. Intrusion Detection System (IDS) is one of the obtainable mechanism that used to sense and classify any abnormal actions. Therefore, the IDS must be always up to date with the latest intruder attacks signatures to preserve confidentiality, integrity and availability of the services. The speed of the IDS is very important issue as well learning the new attacks. This research work illustrates how the Knowledge Discovery and Data Mining (or Knowledge Discovery in Databases) KDD dataset is very handy for testing and evaluating different Machine Learning Techniques. It mainly focuses on the KDD preprocess part in order to prepare a decent and fair experimental data set. The techniques J48, Random Forest, Random Tree, MLP, Naïve Bayes and Bayes Network classifiers have been chosen for this study. It has been proven that the Random forest classifier has achieved the highest accuracy rate for detecting and classifying all KDD dataset attacks, which are of type (DOS, R2L, U2R, and PROBE).
\end{abstract}

Keywords-IDS, DDoS, MLP, Naïve Bayes, Random Forest

\section{Introduction}

Building a reliable network is a very difficult task considering all different possible types of attacks. Nowadays, computer networks and their services are widely used in industry, business and all arenas of life. Security personnel and everyone who has a responsibility for providing protection for a network and its users, have serious con- 
cerns about intruder attacks. Instructive information securing, capacity, coordinated warehousing information examination information mining is utilized as the principle line of rationale [24]

Network administrators and security officers try to provide a protected environment for users' accounts, network resources, personal files and passwords. Attackers may behave in two ways to carry out their attacks on networks; one of these ways is to make a network service unavailable for users or violating personal information. Denial of service (DoS) is one of the most frequent cases representing attacks on network resources and making network services unavailable for their users. There are many types of DoS attacks, and every type has it is own behavior on consuming network resources to achieve the intruder's aim, which is to render the network unavailable for its users [1]. Remote to user (R2L) is one type of computer network attacks, which an intruder sends set of packets to another computer or server over a network where he does not have permission to access as a local user. User to root attacks (U2R) is a second type of attack where the intruder tries to access the network resources as a normal user and after several attempts, the intruder becomes as a full access user [2]. Probing is a third type of attack in which the intruder scans network devices to determine weakness in topology design or some opened ports and then use them in the future for illegal access to personal information. There are many examples that represent probing over a network, such as nmap, portsweep, ipsweep.

Intrusion detection system (IDS) become essential part for building computer network to capture these kinds of attacks in early stages, because IDS works against all intruder attacks. IDS uses classification techniques to make decision about every packet pass through the network whether it is a normal packet or an attack (i.e. DOS, U2R, R2L, PROBE) packet.

Knowledge Discovery in Databases (KDD) is an online repository dataset, which includes all types of intruders' attacks, such as DOS, R2L, U2R, and PROBE. In this report, a number of classifiers will be evaluated on the KDD dataset. The methodology followed in this study is first to perform a preprocessing step on KDD dataset and after that using the prepared dataset on a fair environment and resources. Finally, examining which classifier is more accurate than others in detecting all studied attacks (DOS, R2L, U2R, and PROBE).

The remainder of this work is organized as follows; related work is presented in Section 2, which also provides brief discussion about KDD dataset and selected classifiers. Section 3 gives detailed steps of the preprocessing approach performed on the KDD dataset. The used classification techniques are explained in section 4. Experiments and classifiers evaluation are presented in Section 5. Section 6 presents a comprehensive comparison between the selected classifiers and experimental results with statistical values, followed by conclusions and future work in Section 7.

\section{Related Work}

IDS combines hardware and software to detect attacks on networks in order to ensure the protection of the system from unauthorized access. IDS can be divided into 
two main classification based on the attack's detection method. The first one is the misuse and the second is anomaly detection. The anomaly detection can be used in different ways in order to detect any strange behavior of the user within the network traffic.

IDS built on Artificial Neural Network (ANN) and fuzzy clustering (FC) has been proposed to find out some networks problems and attacks. However, there is limitations of this proposed model for example, it has a lack of accuracy in low-frequent attacks. The researchers here they took over this limitation by dividing heterogeneous training set into homogeneous training subsets, by reducing the complexity of each sub-training set they reduced the complexity, the performance of detection is increased and the backup of the system can be taken successfully by using restore point [3].

Artificial intelligence technique with heuristic algorithm such as Genetic Algorithm (GA) and ANN are used in IDS gaining its ability to learning and development, which makes them more accurate and efficient in facing the increasing number of unpredictable attacks. GA and ANN combined approach gives the IDS with extra performance and accuracy [4].

The assessment of attack impacts, and the streamlining of the system learning structure are issues that s need to continually ponder.[26]

In the work of Pradhan et al [5], they took into account the user actions as a parameter in anomaly detection using a back propagation in their test. Their work very promising. The back propagation neural network had a classification rate of $100 \%$. the detection rate was $88 \%$ on attacks in general whether known or unknown attacks. The main advantage of this work is the minimum amount of trained data that need to give a good results of classification the traffic.

Recently, an improvement alternative of ANN is proposed called Multi-Layer Perception (MLP) ANN. The MLP method made ANN IDS methods more accurate and efficient in terms of detection and normal communication. The MLP-ANN method shows detection result much better than traditional methods. MLP overcomes the limitation of detection low frequency attacks. In addition, MLP-ANN IDS can define the type of attacks and classify them. This feature, allows system to predefine actions against similar future attacks [6] [7] [8].

In the classifier selection model presented by HuyAnh Nguyen and Deokjai Choi [9], they extracted 49,596 instances of KDD dataset and compared a set of classifiers under control environment. Kamlesh Lahreet et al [10] researchers presented different approaches to deal with KDD dataset, supervised and unsupervised methods simulated using matlab, and researchers test supervised and unsupervised techniques with fuzzy rules to identifying performance of proposed system. LEO BREIMAN [11] focused on random forest and how is it combined between trees predictors, researcher proposed error in random forest as limit number of trees in the forest.

Bhargava et al [12] in illustrated in decision tree analysis on j48 algorithm and how it is important to calculate entropy and information gain for each attributes in any dataset ready to be classified, they used decision tree with univariate and multivariate methods also researchers presented multivariate method as linear machine method. The researchers recommended this approach for large amount of data. 
Chris Fleizachet al in [13] stated that nature of dataset sometimes makes it difficult to select appropriate attributes to learn, researchers implement experiments with $\mathrm{Na}$ ivve Bayes classifier and measure performance for each call. The impact of globalization and the rise of developing markets are one of the variables in charge of the expansion experienced by the mold business as of late[25]

\section{$3 \quad$ Knowledge Discovery Dataset Pre-processing}

MIT Lincoln labs provided KDD dataset, it is very helpful to examine which classifier demonstrates high accuracy to detect (DOS, R2L, U2R, and PROBE) attacks. $\mathrm{KDD}$ dataset has imported to Oracle database server, because there was a need to extract fairly experimental dataset for a set of classifiers with statistical information about each type of attack at KDD dataset, also to collect statistical information about each attack type instance. Table 1 illustrates KDD dataset after importing it to the database server and the table lists number of instances for each type of attack.

Table 1. Number of instances for each type of attack

\begin{tabular}{|l|c|}
\hline \multicolumn{1}{|c|}{ Attack Type } & Number of instances \\
\hline SMURF(DOS) & $2,807,886$ \\
\hline NEPTUNE(DOS) & $1,072,017$ \\
\hline Back (DOS) & 2,203 \\
\hline POD (DOS) & 264 \\
\hline Teardrop (DOS) & 979 \\
\hline Buffer overflow (U2R) & 30 \\
\hline Load Module (U2R) & 9 \\
\hline PERL (U2R) & 3 \\
\hline Rootkit (U2R) & 10 \\
\hline FTP Write (R2L) & 8 \\
\hline Guess Passwd (R2L) & 53 \\
\hline IMAP(R2L) & 12 \\
\hline MulitHop (R2L) & 7 \\
\hline PHF (R2L) & 4 \\
\hline SPY (R2L) & 2 \\
\hline Warez client (R2L) & 1,020 \\
\hline Warez Master (R2L) & 20 \\
\hline IPSWEEP (PROBE) & 12,481 \\
\hline NMAP (PROBE) & 2,316 \\
\hline PORTSWEEP(PROBE) & 10,413 \\
\hline SATAN (PROBE) & 15,892 \\
\hline Normal & 972,781 \\
\hline
\end{tabular}

We have 21 types of attacks, categorized into four main groups with different number of instances and occurrences. After extract full KDD dataset, all instances of experiment are full randomized; we have the following table with 148,758 instances organized as follows (Table 2): 
Table 2. Number of Instances after organization

\begin{tabular}{|l|c|}
\hline \multicolumn{1}{|c|}{ Attack Type } & Number of instances \\
\hline SMURF(DOS) & 85,983 \\
\hline NEPTUNE(DOS) & 32,827 \\
\hline Back (DOS) & 70 \\
\hline POD (DOS) & 10 \\
\hline Teardrop (DOS) & 30 \\
\hline Buffer overflow (U2R) & 10 \\
\hline Load Module (U2R) & 2 \\
\hline PERL (U2R) & 1 \\
\hline Rootkit (U2R) & 5 \\
\hline FTP Write (R2L) & 2 \\
\hline Guess Passwd (R2L) & 10 \\
\hline IMAP(R2L) & 4 \\
\hline MulitHop (R2L) & 2 \\
\hline PHF (R2L) & 1 \\
\hline SPY (R2L) & 1 \\
\hline Warez client (R2L) & 31 \\
\hline Warez Master (R2L) & 7 \\
\hline IPSWEEP (PROBE) & 382 \\
\hline NMAP (PROBE) & 70 \\
\hline PORTSWEEP(PROBE) & 318 \\
\hline SATAN (PROBE) & 487 \\
\hline Normal & 28,500 \\
\hline
\end{tabular}

After preparing the KDD dataset for classification experiment techniques, the idea for the next step is to work with the most common used classifier: multilayer perception, Bayesian algorithm, trees and rules using Waikato Environment for Knowledge Analysis (weka) software.

\section{Classification Techniques}

Decision tree first introduced by [14]. It is the most common classifier used to manage database for supervised learning that gives prediction about new unlabeled data, J48 creates Univariate Decision Trees. J48 based used attribute correlation based on entropy and information gain for each attributes [12].It has been used in many fields of study, such as data mining, machine learning, information extraction, pattern recognition, and text mining. It has many advantages; it is capable of dealing with different input data types: numeric, textual and nominal. J48 decision tree is an extension of the algorithm ID3. It has an advantage over ID3 in that it can build small trees. It follows a depth-first strategy, and a divide-and-conquer approach. 


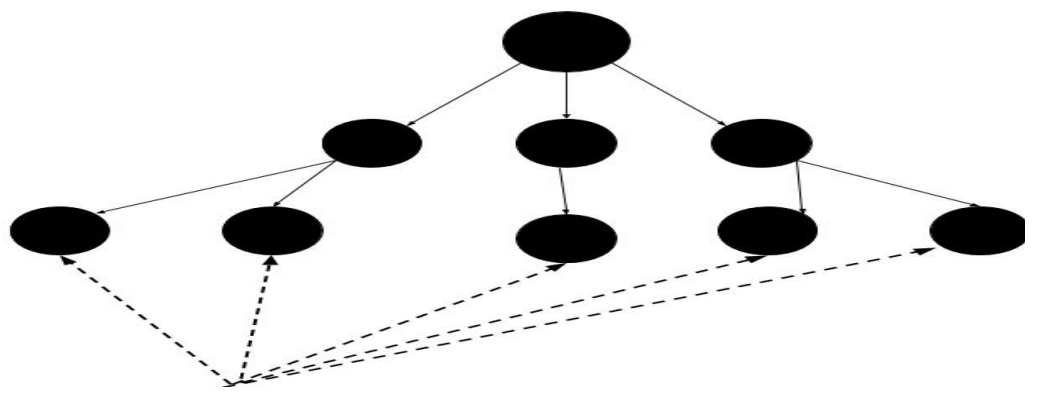

Fig. 1. Decision Tree Structure

A decision tree consists of several elements: root, internal nodes and leaves. The internal nodes represent the conditions in which the value of the parameters will be tested. Based on these values and the condition, the flow of the tree will be decided (along which branch the decision tree must go). Leaf nodes represent the decision or the class. Figure 1 shows a typical decision tree structure.

The tree is constructed by following these three main steps:

- Ensure that all of the grouped inputs are of the same class. Then ensure that the tree is labeled with the class.

- Calculate some parameters for each attribute, such as information gain.

- Choose the best split attribute based on the criteria that have been set.

Entropy comes from information theory; it indicates the amount of information that is held; in other words, the higher the antwon r the more information content there is. It can be measured by:

$$
\text { Entropy }=\quad-\text { pi } \log 2 \mathrm{pi}
$$

Where $\mathrm{Pi}$ is the probability of 1

Information gain expresses the importance of the feature or attribute, and it determines which attribute is the most important one for distinguishing between the classes to be knowledgeable. This piece of information is calculated also on training data. Information gain can help in choosing the best split; if it has a high value then this split is good, otherwise the split is not good enough. Information gain can be calculat-

ed by the data achieved from entropy:

$$
\text { Information Gain = entropy (parent) }- \text { [average entropy }(\text { children })]
$$

\subsection{Random forest}

Classifier was founded by LEO Breiman and Adele Cutler [14], combining tree classifiers to predict new unlabeled data. The predictor depends on a constant that 
denotes the number of trees in the forest; the attributes are selected randomly, and each number of set (trees) here, theu represent a one forest, and each one of these forests represents a prediction class. In this algorithm, random features selection will be selected for each individual tree.

A random forest classifier is an ensemble learning algorithm for classification and prediction of the outputs that is based on an individual number of trees [15]. Using random forest classifiers, many classification trees will be produced, and each separate tree is built by different parts of the general dataset. After each tree classifies an unlabeled class, the new object will be implemented and each tree will vote for a decision. The forest chosen as the winning class is based on the highest number of recorded votes. The number of votes is calculated as follows:

Random forest algorithms:

If there is a dataset, we need to split $\mathrm{n}$ samples from the whole dataset, giving ( $\mathrm{n}$ samples $=$ number of trees).

Each dataset sample needs to be regressed or classified; for each record this is randomly split among all predictor classes to reach an approximately optimal split. Bagging can be learned as a special scenario when $\mathrm{m}$ (tries) $=\mathrm{P}$ (number of predictors).

Predict unlabeled classes based on a reassembled number of aggregation prediction number of trees.

The accuracy rate and error rate for Random Forest are the tuning parameters for Random forest (RF) classifiers can be measured either by splitting the whole dataset, for example by testing $40 \%$ and for training $60 \%$, or by dividing the data into 10 s or 20 s, etc. After a random forest is built the test model with $40 \%$ of the data can be used to calculate error rate, and accuracy rate can be measured based on comparisons of correctly classified instances with incorrectly classified instances.

Out of the bag (OOG) is another way of calculating the error rate in this technique; there is no need to split the dataset because calculation occurs within the training phase. The following parameters need to be adjusted correctly to reach the highest accuracy rate with a minimum error rate:

- Number of trees.

- The number of descriptors that occur randomly for present candidate's m (tries).

After analyzing and studying many cases, 500 trees are needed within the descriptor that may be desired. Even if there are great numbers of trees that will not achieve the highest accuracy rate, except for wasting training time and resources [16], random forest tuning parameters are represent a hot research area that needs to be fine-tuned. Figure 2 shows random forest architecture: 


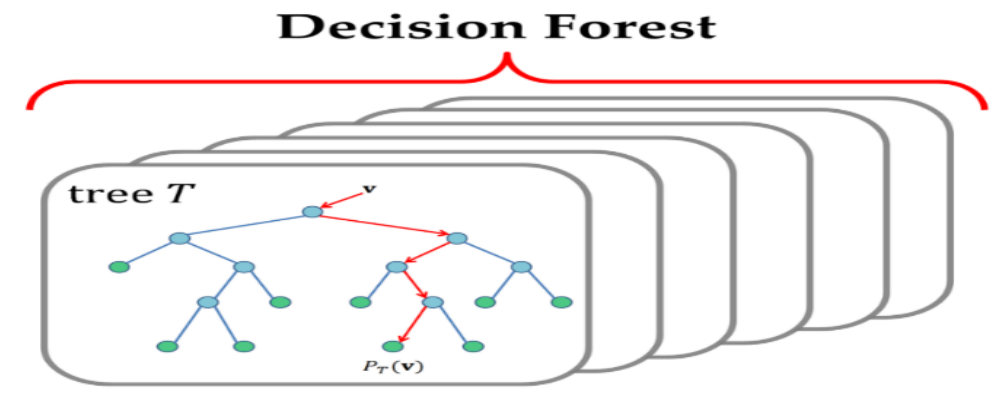

Fig. 2. Random Forest Architecture [13].

\subsection{Multilayer Perceptron (MLP)}

MLP is widely use neural network classifier based on number of classes (output) and number of hidden layers, MLP uses weights for every node at neural network most effective attributes will get large weights conversely attributes not affect in predictive class. MLP always takes largest time for training but it has quick time for testing [17]. MLP has been positively used in daily life uses, like; regression problems, classification and prediction problems.

An example of a modest structure MLP network is illuminated in Figure 4. MLP drive the data flow to be taken in one direction from input to output. As there will be no feedback; According to [18] and [19], any MLP network can be notable by a number of performance features, which can be brief in three points:

Neural Network Architecture: Overall, MLP architecture can be clarified as set of links between the neurons in different layers. Generally, the architecture consists of three main layers: input layer, hidden layers and output layer. MLP is most of the time fully connected. On each link there is a weight, which is tuned based on the training algorithm.

Training Algorithm: Is the method of selecting one model from a set of models, which tunes the weights of the links. Table 3 illustrates examples of some common transfer functions.

Transfer Function: Is applied on the net input of each neuron to control the net output signal. Here in, the function is usually non-linear. The most common function used as transfer function is Sigmoid function. The use of the sigmoid function has an advantage in neural networks trained by a back propagation learning algorithm. The sigmoid function and other common transfer functions are used as shown in Figure 3.

To understand how the learning process on MLP is done, here is a simple example to demonstrate the process, suppose that we have an MLP, which has $\mathrm{N}$ neurons as input layer and $\mathrm{M}$ neurons in the hidden layers, and single output neuron. The learning process will as follow: 
Table 3. Transfer Functions [15].

\begin{tabular}{|c|c|}
\hline Transfer function & definition \\
\hline \hline Linear & $f(x)=x$ \\
\hline Sigmoid & $f(x)=\frac{1}{1+e^{-x}}$ \\
\hline Hyperbolic & $f(x)= \begin{cases}0, & x<0 \\
1, & x \geq 0\end{cases}$ \\
\hline Hard limit & $f(x)= \begin{cases}-1, & x<0 \\
1, & x \geq 0\end{cases}$ \\
\hline
\end{tabular}

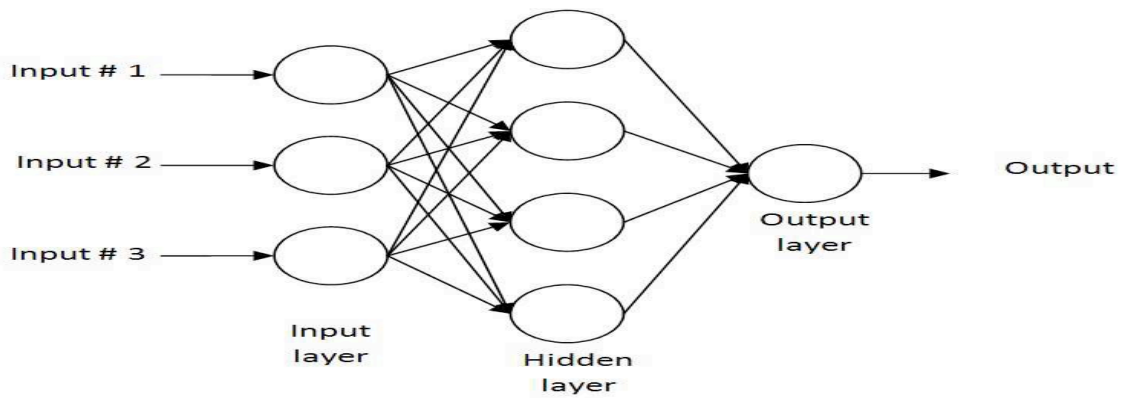

Fig. 3. MPL Architecture

Hidden Layer Stage: Given a number of inputs I (the output of the input layer) and a set of equivalent weights as also an input between the input and hidden neurons wij, then the outputs of all neurons in the hidden layer are calculated as in Equation (3) and Equation (4):

$$
\begin{gathered}
O_{i}=\sum_{i=0}^{N} w i j \psi i \\
y_{j}=z\left(O_{j}\right)
\end{gathered}
$$

where $\mathrm{i}=1,2 \ldots, \mathrm{N}$ and $\mathrm{j}=1,2 \ldots \mathrm{M}$. The $\mathrm{z}$ and $y j$ are the activation function and output of the $j^{\text {th }}$ node in the hidden layer, respectively. The $\mathrm{z}$ is usually a sigmoid function which given in Equation 5.

$$
z(x)=\frac{1}{1+e^{-x}}
$$

Output stage: Equation (6) is the final outputs of all neurons in the output layer. For simplicity the equation bellow explain the output :

$$
Y^{\wedge}=f\left(\sum_{j=0}^{m} w_{j} y_{j}^{H}\right)
$$

Where $f()$ is the activation function of the output layer, which is typically a linear function. And the $Y^{\wedge}$ is the output of the neural network. The MLP network is always 
trying to make the error very small through the Back Propagation (BP) Training algorithm. At the beginning, all the weights initialized with a random values, and after that the weights are changing in each iteration until satisfied state values are obtained.

Error Validation Stage: ANN keeps learning until the error becomes very small assuming that the observed output is $\mathrm{Y}$ and the predicted output is ${ }^{\wedge} \mathrm{Y}$. The learning process will keep going until the error difference given in Equation (7) is a minimum value, as the minimum is the best. $\mathrm{N}$ is the total number of instances that used during training stage.

$$
\text { Error }=\frac{1}{N} \sum_{i=1}^{T}\left(Y_{i}-Y_{i}\right)^{2}
$$

In MLP, the weights and bias values are allocated randomly, here in, the goal of the training is to find the set of weights that give the output of the network to be close as possible to the real values.

\subsection{Naïve Bayes}

It is a simple probabilistic classifier that returns $\mathrm{p}(\mathrm{y} \mid \mathrm{x})$, and calculates probabilistic for each class in a dataset and defines discriminative learning to predict the values of the new class. More about the main formulation for Nave Bayes may be found in [18].

A Naïve classifier links the dataset attributes $x \in X$ that are used as inputs to the class labels $Z \in\{1,2, C\}$, where $X$ is the attribute space and $Z$ is the class space. Let $\mathrm{X}=\mathrm{IRD}$ where $\mathrm{D}$ is a real number. The Naïve classifier may be used with discrete and continuous attributes. This model is called a multi-label problem. The learning function that directly computes class $p=\left(\frac{y}{x}\right)$ is called a discriminates model. The main aim is to learn the conditional class that is used for non-linear and multi-label problems. For this reason we will use Equation (8):

$$
p(y \mid x)=\frac{p(x, y)}{p(x)}=\frac{p\left(\frac{x}{y}\right) p(y)}{\sum_{y^{\prime}=1}^{c} \frac{p\left(\frac{x}{y^{\prime}}\right)}{p(y)}}
$$

The Naïve classifier achieves outputs based on an argument max function that is shown in Equation (9):

$$
f(x)=y^{\prime}(x)=\operatorname{argmax}\left\{\left(p\left(\frac{y}{x}\right)\right)\right\}
$$

Probabilistic classifiers have the following advantages in [18]:

- Option to reject which is used when we are uncertain of the prediction result, so the prediction result can be ignored since human effort exists.

- Allow learning function to be changed and a combination of probability functions can be used to reach highest performance. The main issues are if the direct learning 
function $p=\left(\frac{y}{x}\right)$ is used and the probability function is changed; there is no need to recalculate $=\left(\frac{y}{x}\right)$.

- Balanced classes of some of the collected datasets have unbalanced classes which means that if we have one million records of normal network traffic where there is only 1 abnormal for 1000 records we can directly train the unbalanced training dataset and easily achieve an accuracy rate of $99 \%$ by just using class always $=$ normal. To handle such problem-balanced classes, Equation (10) and Equation (11) are used.

$$
\begin{gathered}
\operatorname{Pbal}(y \mid x) \propto=P(x, y) \operatorname{Pbal}(y) \\
\operatorname{Ptrue}(y \mid x) \propto \operatorname{Ptrue}(x, y) \operatorname{Ptrue}(y) \propto \frac{P\left(\frac{y}{x}\right)}{\operatorname{Pbal}(y)} \operatorname{Ptrue}(y)
\end{gathered}
$$

Model combinations are very useful when the collected dataset contains a mix of feature types, such as if there is a collected dataset and each feature vector represents a distinguished data type (text, images, numbers, etc.) Two or more kinds of attributes using model combinations can build two or more classifiers, such as $p\left(\frac{y}{x_{1}}\right) \cdot p\left(\frac{y}{x 2}\right)$ and so on (Murphy, 2006). To combine two different information sources, Equation (12) is used:

$$
P(x 1, x 2 \mid x y)=P(x 1 / y) P(x 2 / y)
$$

\subsection{Bayes Network}

It is a classifier for supervised learning that uses assumptions of independent features. It uses theory of learning that represents distribution naïve Bayesian classifier. It uses various search algorithms and different quality measure methods. Bayes Network is an enhancement for Naïve Bayes [19].

A Bayesian network is very useful, because it helps us to understand the world we are modeling. BayesNet may be the best in various areas of life, where modeling a mysterious fact and in the state of decision nets, wherever it is good to make intelligent, justifiable and quantifiable decisions that will enhance performance of classification. In brief, BayesNet is helpful for diagnosis, prediction, modeling, monitoring and classification [20].

The main idea of the Bayesian classifier consists of two phases: in the first, if an agent has an idea and knows the class, in this case it can predict the values of the other features; in the second, if the agent does not have an idea or does not know the class, in this case the Bayes rule is used to predict the class given.

We used the Bayesian Network as a classifier for the following reasons:

- Probabilistic learning, which calculates clear probabilities for assumption.

- Incremental, which is a prior knowledge and possible to be added to data viewing.

- Probabilistic prediction, which can predict more than one hypothesis, weighted by the probabilities. 
The theory of the Bayesian Network is shown in Equation (13), where the symbol $\mathrm{D}$ indicates the training data, the probability of hypothesis $h$.

$$
P(h \mid D)=\frac{P(D \mid h) P(h)}{P(D)}
$$

The symbols in Equation 4 refer to:

$\mathrm{P}(\mathrm{h} \mid \mathrm{D})$ : posterior probability.

$\mathrm{P}(\mathrm{D} \mid \mathrm{h})$ : condition probability.

$P(h)$ : prior probability of $h$.

$\mathrm{P}$ (D): marginal probability of $\mathrm{D}$.

\section{Performance Evaluation of the Selected Classifiers}

KDD dataset presents real packets focused on wired network; it has 41 features about each packet that will help to implement different classifier types. The current experiments that are performed present fair test environment because we extracted 148,758 instances from all four groups of attack (DOS, R2L, U2R, and PROBE) as training dataset, normal packets present \%19 from current experiment as original KDD dataset normal packets and the highest proportion for the DOS attack with 79\% from current experiment as original KDD dataset DOS packets.

For fair control comparison between different classifiers, another 60,000 independent instances were extracted from original KDD dataset as test sample and these instances fully randomized and not included in training dataset. The experiment environment applied with Weka version 3.7.12 and Intel Xeon (R) CPU E5-2680@ 2.70GHzX4 with available RAM 8.0GB under Ubuntu 13.10 platform. Most common classifiers are used in this experiment (J48, Random forest, Random Tree, Decision Table, Multilayer Perceptron (MLP), Naïve Bayes and Bayes Network). All models and results are saved to start comprehensive study about which classifier has the highest accuracy rate to detect attacks.

\section{Experiment Evaluation and Results}

All selected classifiers tested with 60,000 independent instances from KDD dataset and all test instances are fully randomized. This section illustrates all parameters values that have been used in selected classifiers in the experiments.

J48 tree classifier has been tested with the parameters below:

Confidence factor $=0.25$; numFolds $=3$; seed $=1$; unpruned $=$ False, collapse tree $=$ true, and sub tree rising $=$ true. Random forest classifier also tested with the following parameters: Number of trees $=100$ and seed $=1$.

Random tree classifier was tested with the following parameters: Min variance = 0.001 and seed $=1$. Decision table classifier was tested with the following parameters: Search techniques best first and cross value $=1$. Multilayer Perceptron (MLP) classi- 
fier was tested with the following parameters: Search learning rate $=0.3$, momentum $=0.2$ and validation threshold $=20$. Bayes Network classifier was tested with the following parameters: Search techniques estimator value $=$ simple estimator and search technique $=\mathrm{K} 2$ algorithm.

Table 4 lists statistical values that achieved in our experiments and it can be seen that random forest classifier achieves the highest Kappa statistic with rate equals to 0.8957 and the lowest Kappa statistic with Bayes network classifier with rate eqauals to 0.8464 .

Table 5 records weighted average for true positive (TP) and false positive ( FP) for each classifier selected for experiment, also the random forest achieves the highest TP rate with value equals to 0.938 .

Table 6 presents accuracy rate that recorded in the experiment. The random forest classifier achieves the highest rate accuracy.

Table 4. Statistical Values

\begin{tabular}{|l|c|c|c|}
\hline \multicolumn{1}{|c|}{ Classic } & Kappa Statistic & Mean Absolute Error & $\begin{array}{c}\text { Root Mean Square } \\
\text { Error }\end{array}$ \\
\hline J48 & 0.8844 & 0.0059 & 0.0763 \\
\hline Random Forest & 0.8957 & 0.01 & 0.0682 \\
\hline MLP & 0.8639 & 0.0075 & 0.0813 \\
\hline Naïve Bayes & 0.8542 & 0.0076 & 0.0872 \\
\hline Bayes Network & 0.8464 & 0.0085 & 0.087 \\
\hline
\end{tabular}

Table 5. Weighted average for true positive(TP) and false positive ( FP)

\begin{tabular}{|l|c|c|c|c|}
\hline \multicolumn{1}{|c|}{ Classifier } & TP Rate & FP Rate & Precision & ROC Area \\
\hline J48 & 0.931 & 0.005 & 0.989 & 0.969 \\
\hline Random forest & 0.938 & 0.001 & 0.991 & 0.996 \\
\hline Random tree & 0.906 & 0.001 & 0.992 & 0.953 \\
\hline MLP & 0.919 & 0.014 & 0.978 & 0.990 \\
\hline Naïve Bayes & 0.912 & 0.002 & 0.988 & 0.969 \\
\hline
\end{tabular}

Table 6. Accuracy rate

\begin{tabular}{|l|c|c|c|}
\hline \multicolumn{1}{|c|}{ Classifier } & $\begin{array}{c}\text { Correctly classified In- } \\
\text { stances }\end{array}$ & $\begin{array}{c}\text { incorrectly classified In- } \\
\text { stances }\end{array}$ & Accuracy \\
\hline J48 & 55865 & 4135 & $93.1083 \%$ \\
\hline Random Forest & 56265 & 3735 & $93.775 \%$ \\
\hline Random tree & 54345 & 5655 & $90.575 \%$ \\
\hline MLP & 55141 & 4859 & $91.9017 \%$ \\
\hline Naïve Bayes & 54741 & 5259 & $91.235 \%$ \\
\hline Bayes Network & 54439 & 5561 & $90.7317 \%$ \\
\hline
\end{tabular}

\section{Conclusions and Future Work}

Due to the urgent demand for an effective IDS in network security, researchers are striving to identify improved approaches. This research work illustrates how the KDD dataset is very useful for testing different classifiers. The work concentrates on KDD preprocess phase to prepare fair experiments and fully randomized independent test data. Among the classification techniques (J48, Random Forest, Random Tree, Deci- 
sion Table, MLP, Naïve Bayes, and Bayes Network), the Random Forest classifier has achieved the highest accuracy rate for detecting and classifying all KDD dataset attack types (DOS, R2L, U2R, and PROBE). KDD dataset has 41 attributes and all of them have been recorded, but as part of future work a data engineering phase is going to be added to the study that will focus on which attribute has an essential role in achieving the highest accuracy for selected classifiers in our experiments.

\section{$8 \quad$ References}

[1] N. Huy and C. Deokjai, "Application of data mining to network intrusion detection: classifier selection model," Challenges for Next Generation Network Operations and Service Management, pp. 399--408, 2008.

[2] S. Paliwal and R. Gupta, "Denial-of-service, probing \& remote to user (R2L) attack detection using genetic algorithm," International Journal of Computer Applications, vol. 60 , no. 19 , pp. 57--62, 2012.

[3] D. Gaikwad, S. Jagtap, K. Thakare and V. Budhawant, "Anomaly based intrusion detection system using artificial neural network and fuzzy clustering," International Journal of Engineering\}, vol. 1, no. 9, 2012.

[4] V. Bapuji, R. N. Kumar, A. Goverdan and S. Sharma, "Soft computing and artificial intelligence techniques for intrusion detection system," Networks and Complex Systems, vol. 2, no. 4, 2012.

[5] M. Pradhan, S. K. Pradhan and S. K. Sahu, "Anomaly detection using artificial neural network," International Journal of Engineering Sciences \& Emerging Technologies, vol. 2, no. 1, pp. 29--36, 2012.

[6] M. Sammany, M. Sharawi, M. El-Beltagy and I. Saroit, "Artificial neural networks architecture for intrusion detection systems and classification of attacks," in The 5th international conference INFO2007, 2007.

[7] M. Al-Kasassbeh, G. Al-Naymat, A. Hassanat and M. Almseidin, "DetectingDistributed Denial of Service Attacks Using Data Mining Techniques," International Journal of Advanced Computer Science and Applications, vol. 7, no. 1, 2016.

[8] M. Al-kasassbeh, "An Empirical Evaluation For The Intrusion Detection Features Based On Machine Learning And Feature Selection Methods," Journal of Theoretical and Applied Information Technology, vol. 22, p. 95, 2017.

[9] H. Nguyen and D. Choi, "Application of data mining to network intrusion detection:classifier selection model," Challenges for Next Generation Network Operations and Service Management, pp. 399--408, 2008.

[10] M. K. Lahre, M. T. Dhar, D. Suresh, K. Kashyap and P. Agrawal, "Analyze different approaches for IDS using KDD 99 data set," International Journal on Recent and Innovation Trends in Computing and Communication, vol. 1, no. 8, pp. 645--651, 2013.

[11] L. Breiman, "Random forests," Machine learning, vol. 45, no. 1, pp. 5--32, 2001. https://doi.org/10.1023/A:1010933404324

[12] N. Bhargava, G. Sharma, R. Bhargava and M. Mathuria, "Decision tree analysis on j48 algorithm for data mining," Proceedings of International Journal of Advanced Research in Computer Science and Software Engineering, vol. 3, no. 6, 2013.

[13] C. Fleizach and S. Fukushima, A naive Bayes classifier on 1998 KDD Cup, echnical Report, Department of Computer Science and Engineering, University of California,San Diego, 1998.

[14] Breiman, Leo, Friedman, J. H, Olshen, R. A, Stone and C. J, "Classification and regression trees. Wadsworth," Belmont, CA, 1984. 
Paper-Intensive Pre-Processing of KDD Cup 99 for Network Intrusion Classification...

[15] A. Araar and R. Bouslama, "A comparative study of classification models for detectionin ip networks intrusions," Journal of Theoretical \& Applied Information Technology, vol. 64, no. $1,2014$.

[16] M. A. M. Hasan, M. Nasser, B. Pal and S. Ahmad, "Support vector machine andrandom forest modeling for intrusion detection system (IDS)," Journal of IntelligentLearning Systems and Applications, vol. 6, no. 1, p. 45, 2014. https://doi.org/10.4236/ji lsa.2014.61005

[17] S. K. Pal and S. Mitra, "Multilayer perceptron, fuzzy sets, and classification," IEEE Transactions on neural networks, vol. 3, no. 5, pp. 683--697, 1992. https://doi.org/10.1 $109 / 72.159058$

[18] K. Gurney,introduction to neural networks, CRC press, 1997

[19] L. Fausett and L. Fausett, Fundamentals of neural networks: architectures, algorithms, and applications, Prentice-Hall, 1994.

[20] J. Jorgenson, C. a. Manikopoulos, J. Li and Z. Zhang, "A hierarchical AnomalyNetwork Intrusion Detection System Using Neural Network Classification," in Workshop on Information, 2001.

[21] N. Friedman, D. Geiger and M. Goldszmidt, "Bayesian network classifiers," Machine learning\}, vol. 29, no. 3, pp. 131--163, 1997. https://doi.org/10.1023/A:1007465528199

[22] S. B. Kotsiantis, I. Zaharakis and P. Pintelas, Supervised machine learning: A review of classification techniques, 2007.

[23] M. Alkasassbeh and M. Adda, "Network fault detection with Wiener filter-based agent," Journal of Network and Computer Applications, vol. 32, no. 4, p. 824-833, 2009. https://doi.org/10.1016/j.jnca.2009.02.001

[24] Y. Zhu "A Data Driven Educational Decision Support System," international journal of emerging technologies in learning, Vol 13, No 11 (2018)

[25] Z. [26] J. Chen, J. Zhao, "An Educational Data Mining Model for Supervision of Network Learning Process," International Journal of Emerging Technologies in Learning Vol 13, No 11 (2018)

[26] Fabiola González Romo! , "Storytelling and Social Networking as Tools for Digital and Mobile Marketing of Luxury Fashion Brands," International Journal of Interactive Mobile Technologies, Vol 11, No 6 (2017)

\section{Authors}

Ibrahim M. Obeidat is an associate Professor, working in Prince Al-Hussein Bin Abdullah II Faculty of Information Technology; Hashemite University, Jordan since 2008, his interest is in networking and cyber security

Mouhammd Alkasassbeh is an associate Professor, working in computer science department, Princess Sumaya University for technology; his interest is in networking and security.

Nabhan Hamadneh is an assistance Professor working in Prince Al-Hussein Bin Abdullah II Faculty of Information Technology, Hashemite University, his interest is in networking.

Mohammad Almseidin is a PhD candidate at the Department of Information Technology, University of Miskolc, his interest is in networking and security

Mazen Ibrahim AIZubi is Senior cyber security engineer Working for ALDAR Financial brokerage since 2005. Hold a master degree in Network security specialization in Cyber Security from Zayed university located in Abu Dhabi -UAE

Article submitted 09 October 2018. Resubmitted 26 November 2018. Final acceptance 330 November 2018. Final version published as submitted by the authors. 